\title{
Therapeutische Umschau
}

Einige neuere Erfahrungen über die Behandlung des Trachoms mittels der Excision. Von Dr. Blumenthal (Riga). Beiträge zur Augenheilk., herausgegeb. von Deutschmann. 73. Heffc.

Der Verfasser, der sich als überzeugten Anhänger des Excisions-verfahrens in der Behandlung des Trachoms bekennt, bespricht an der Hand seiner in mehr als 15 Jahren gesammelten Erfahrungen, die sich auf über 4000 Operierte erstrecken, insbesondere die Fragen, ob der primäre Erfolg immer um so günstiger sei, wie er ihn im allgemeinen von dieser Operation bei Trachom in jeder Form gesehen habe, dann, ob der günstige Erfolg von Dauer sei, und endlich, ob nicht die Operation in manchen Fallen auch Schaden stifte.

Die erste Frage wird entschieden bejaht, vorausgesetzt, dass die Excision in einem Krankheitsstadium ausgeführt worden sei, wo über`» haupt noch etwas zu excidieren gewesen sei. Doch sei dieses Stadium oft ein sehr lange andauerndes, das sich über Dezennien erstrecken könne. Er halt es hinsichtlich des Erfolges für wichtig, dass auch von der Konjunktiva des unteren Lides möghchst viel von der tracho-matösen Wucherung, erforderlichenfalls auch ein breiterer Streifen von Knorpel mit excidiert werde, im Gegensatz besonders zu Kuhnt, der am unteren Lide eine Excision von Knorpel gar nicht oder nur in geringer Ausdehnung gestattet. Nicht recht zu verstehen ist die Warnung des Verfassers vor der subkonjunktivalen Injektion von Kokain, die er als Ursache von bedrohlichen Nachblutungen, von Wundeiterung und verzögerter Heilung bezeichnet, und der er die Schuld beimessen zu sollen glaubt, dass die Reinigung der zurück-bleibenden Teile der Conjunctiva Tarsi sich verzögere oder ganz stocke, was nach ihm wahrscheinlich auf die gewebstötende Eigenschaft des Kokains zurückzuführen sei.

Was die Frage nach dem Dauererfolg der Excision anlangt, so ist Verfasser der Überzeugung, dass Rezidive nur dann erfolgen, wenn die Operation resp. die Nachbehandlung nicht genügend sorgfältig geleitet worden ist. Besondere Aufmerksamkeit ist, wie schon Kuhnt betonte, darauf zu verwenden, die nach der Operation etwa zurück-gebliebenen Reste trachomatöser Herde, die sich besonders oft im inneren, aber auch im äusseren Lidwinkel finden, nachträglich, d. h. nach Abklingen der Wundreaktion von der ersten Operation, fortzu-schaffen. Mit dieser Nachoperation wird zweckmässig auch eine Ab-schabung der eventl. noch vorhandenen, infolge der Wundreaktion neu aufgetretenen feinen sandkornförmigen Granulationen der Konjunktiva mit dem scharfen Löffel verbunden. Ebenso sind eventuelle Pannusreste auf der Kornea gründlich abzuschaben. Zur Unterstützung der Resorption des Pannus empfiehlt er durch längere Zeit fortgesetzte Adrenalineinträufehmgen. Ist im Verlaufe etwa eines halben Jahres nach der Operation, bezw. nach günstigem Abschluss der Nachbehandlung kein Rezidiv erfolgt, so sind die Patienten mit grösster Wahr-scheinlichkeit vor späteren Rezidiven gesichert.

Die schliessliche Frage nach etvvaigen Nachteilen infolge der Ope376

Therapeutische Umschau. ration beantwortet Verfasser dahin, dass bisweilen danach Ptosis ein-trete (nach seineni Material etwa in 1 pCt. der Fälle), am häufigsten noch nach der Operation bei Kíndern, und überhapt nach der Operation von frischen Trachomfällen. In frischen Fallen solle man daher nicht übermässig 
grosse Stücke ausschneiden, besonders nicht im äusseren, am wenigsten im inneren Augenwinkel. Jedenfalls 1.asse sich aber die Ptosis nach der Operation mit seltenen Ausnahmen vermeiden; sie zeige, wo doch eingetreten, meist nur einen geringen Grad, und sei schhesslich unschwer zu beseitigen, wobei er der Fadenoperation nach Pagenstecher den Vorzug gibt. Die Behauptung, dass nach der Excision Xerosis entstehe, wurde bekanntlich schon von Kuhnt zurückgevviesen, auch der Verfasser weist sie als vollkommen unbegründet zurück, Im Gegenteil wird durch korrekte Ausführung der Operation und sorgfältige Nachbehand-lung dem Eintreten der Xerose geradezu vorgebeugt.

Die Infektionsverhütung bei Bulbusoperationen. Von Prof. Elschnig (Prag). Mediz. Klinik 1909, No. 38.

Verfasser betont, dass die Quelle der postoperativen Infektionen im Bindehautsack selbst zu suchen sei, dass somit die Prophylaxe der postoperativen Infektionen in der entsprechenden Beachtung des Bakteriengehaltes der Bindehaut gipfle. Exakte bakteriologische Untersuchungen haben ihm gezeigt, dass alle Infektionen bei Star-operationen durch Streptokokken bezw. Pneumokokken erzeugt waren, die er bei der bakteriologischen Untersuchung des Bindehautsackes vor Staroperationen in fast 30 pCt. der Fälle fand, und zu deren sicherem Nachweis flüssige Xährböden (Serumbouillon) erforderlich sind. Ergibt eine derartige Kultur, was nach 24 bis längstens 36 Stunden möglich ist, das Vorhandensein dieser oder anderer allgemein pathogener Mikro-organismen, so hat die Operation zu unterbleiben und ist die Bindehaut in geeigneter Weise zu behandeln. Xach 4 bis 8 Tagen wird dann in gleicher Weise eine erneute bakteriologische Kontrolle vorgenommen, und unter keinen Umständen eher operiert, bevor nicht die früher vorhanden gewesenen Streptokokken aus dem Bindehautsacke ver-schwunden sind.

Zusammenfassend betont Elschnig, dass vor Ausführung von grösseren, denBulbus eröffnenden Operationen, also namentlich Lappen-extraktionen, folgende Umstände Berücksichtigung zu finden haben:

normale Beschaffenheit der Lidhaut und der Tränenwege.

Fehlen gröberer entzündlicher Veränderungen der Bindehaut.

Fehlen pathogener Mikroorganismen, vornehmhch von Streptokokken und Pneumokokken im Bindehautsacke (Nachweis durch Kultur auf Serumbouillon).

Fehlen von infektiösen Allgemein- und Organerkrankungen, ins-besondere auch von Angina (Gefahr der endogenen Infektion!).

Bei vorhandenen Stoffwechselerkrankungen (Autointoxikationen) entsprechende Vorbehandlung des Kranken.

Bezüghch der operativen Technik: alle den Bulbus eröffnenden Wunden sollen subkonjunktival gelegen sein, bez $\Lambda \mathrm{v}$. bei der Umschnei-dung eines Lappens ein deckender Bindehautlappen mit umschnitten werden.

Diagnostisch $\theta$ Notizen.

377

Über chronisehe Aderhautentzündung (Chorioiditis disseminata) auf tuber-kuloser Orundlage. Von Prof. Bernheimer (Innsbruck). Ibid.

Unter Hinweis darauf, dass es viel häufiger, als gewölmlicb an-genommen werde, gelinge, die Tuberkulose als latentes ätiologisches Moment bei chronischer disseminierter Chorioiditis festzustellen, be-richtet Verfasser, dass er in den letzten Jahren in mehreren Fallen chronisehe Erkrankungen der Aderhaut unter dem Bilde der Chorioiditis disseminata, ohne dass sonst 
deutliehe Zeichen von Tuberkulose klinisch nachweisbar gewesen wären, durch Tuberkulinkuren der Heilung zu-führen konnte.

Da das ophthalmoskopische Bild in solchen Fallen nichts charak-teristisches bietet und klinisch nachweisbare Zeichen von Tuberkulose meist fehlen, ist zunächst die tuberkulose Natur der Aderhautentzündung festzustellen. Ist durch die Wassermannsche Reaktion das Vorliegen luetischer Infektion unwahrscheinlich gemacht und fällt eine gründliche interne Untersuchung (Blutbefund! Anaemie, Chlorose!) negativ aus, so wird eine Probeinjektion mit Alttuberkulin vorgenommen und bei positivem Ausfall die Tuberkulinkur eingeleitet.

Als wirksamstes Mittel, das gut vertragen wird, bezeichnet Verfasser in Übereinstimmung mit A. v. Hippel die ifoc $\Lambda$ sche Bazülen-emulsíon. Es wird empfohlen, mit kleinen Dosen (1/10oo B.E.) zu be-ginnen und langsam zu steigern. (cf. hierzu auch das Referat über die Arbeit von Davids, diese Zeitschr. XX. Bd., p. 588). Sorgfältige Be-achtung der Temperaturschwankungen! Bei Zunahme der Temperatur über $38^{\circ}$ Pause bis zu 8 Tagen! Behandlung nicht zu früh abbrechen, um Rückfälle zu vermeiden!

Verfasser konnte so fünf Fälle von chronischer Chorioiditis auftuberkulöser Grundlage der Heilung zuführen, die bis jetzt angehaltenhat. In alien Fallen war nach durchgeführter Tuberkulinkur auchein besseres Aussehen und vollkommenes Wohlbefinden der Krankenzu konstatieren. Prof. Dr. $\mathrm{i}^{1 / 1} \mathrm{8}^{1 / 8-B o n n}$.

Diagnostisehe Notizen.

Zur Differentialdiagnose zwischen okularen Kopfschmerzen und solchen, verursacht von Nebenhöhlenentzündungen.

Angeregt durch die bekannte Mitteilung von Axenfeld: ,,Die Be-teiligung des Ophthalmologen an der operativen Behandlung der orbi-talen Xebenhöhlen"1) gibt Snydacker, Augen- und Ohrenarzt an ver-schiedenen Spitälern Chicagos, einige interessante Zahlen aus seiner Privatpraxis:

Bei 2000 Kranken war in 799 Fallen (= ca. 40 pCt. der Patienten) wesentlich „Kopfschmerz” die Ursache, welche die Kranken zu ihm führte, bezw. Überweisung von anderen Ärzten verursachte.

I. In 55 (= 7 pCt. der Fälle)

bei denen Kopfschmerz geklagt $\Lambda$ vurde

war sicher festzustellen

dass kein Augenleiden

sondern Entzündung der Nebenhöhlen der Nase die Ursache der Beschwerden war.

!) Klinische Monatsblätter f. Augenheilkunde 1908

S. 506. 